\title{
Analysis of the Spatio-Temporal Parameters of an Amputee Patient vs. a Healthy Patient
}

\author{
Brigette Paola Torres Bello, Yuliana Ruiz Piragauta, Esperanza Camargo Casallas \\ Universidad Distrital Francisco José de Caldas \\ Bogota, Colombia \\ bptorresb@correo.udistrital.edu.co; yruizp@correo.udistrital.edu.co; ecamargoc@udistrital.edu.co
}

\begin{abstract}
The purpose of this article is to evaluate the spatio-temporal parameters of an amputee versus a healthy patient, since it is a way of recognizing the failures and observations of the gait cycle as a result of the state of the prosthesis.

For this study it is necessary to deepen the biomechanics of the lower extremities by investigating the similarity that exists between a prosthesis and the biological part of the amputated joint. The acquired data were taken from the sagittal and frontal plane, in an orthopedic and rehabilitation center for both types of patients (Transtibial Amputee and Person with intact extremities), these data were processed using inertial sensors of TechnNaid® equipment and taken to a MarchaHumanaET software, which provides the values of the spatio-temporal gait parameters for each subject.
\end{abstract}

Keywords: Human gait, biomedical, spatio-temporal variables, amputee, prosthesis

\section{Introduction}

Inquiring about the functionalities of the human body is a fact of great interest; it is a continuous process of evolution. For some reason we are the most developed species, our anatomy, mechanics and evolution of each part of our body guaranteed our role. The Human Biomechanics is the explanation of our complex system and why we are the only bipedal beings, the clarification of each movement, the strength and determination of each muscle in the gait process.

When performing the biomedical in an articulation the plane of reference, involved joints, the walked degrees and times, are indispensable factors that must be studied in this alternating locomotor process both in people with their intact extremities as amputees. Since it allows an analysis concerning the correct functionality of the prosthesis.

In the sagittal plane the flexion - extension movements are performed, in the frontal plane abduction - adduction and in the axial plane the movements of internal and external rotation. (Clinical model according to Thompson and Floyd, 1997)

The kinematic analysis provides the detail of each joint at the moment in which the gait begins without taking into account the forces that are made, from the kinetic analysis we can find the mathematical observation during each phase. We also have the spatio-temporal variables to study the step length, the time in which the cycle is performed during the swing and support phases, the cadence of the steps in a spontaneous speed, in addition to the speeds in each of the phases of the cycle. [1]

In this article we analyze the spatio-temporal parameters of the gait of a transtibial amputee vs. a healthy patient in the sagittal and frontal plane. The study was performed on a patient with a unilateral transtibial prosthesis in an orthopedic and rehabilitation center and a patient with intact extremities adding similar body characteristics, allowing to compare the gait process. The equipment used for this analysis is the TechNaid® device; a system of motion capture that analyzes the biomechanics of the human body through the angles formed between joints, this equipment has a system of sensors that allow obtaining kinematic results and other variables (angular velocity, acceleration, magnetic field and temperature) through a software called "Tech-MCS Studio 4". These data are taken to a software called "Human March ET" developed by the Research Group DIGITI and the District University, which presents the spatio-temporal variables for a more complete analysis of the gait.

\section{Materials and Methods}

Tech Naid ${ }^{\circledR}$ is a system of capture of the human body. It contains inertial sensors called Tech IMU CV4 which measure acceleration and angular velocity, responsible for the capture system which contain gyroscopes, accelerometers and magnetometers. The data output can be generated via USB port or Bluetooth communication with 
an indoor capture distance of $50 \mathrm{~m}$ and outdoor $150 \mathrm{~m}$. It obtains data like: acceleration, angular velocity, magnetic field and joint angles.

Tech-MCS Studio 4 is the software that complements Tech MCS. It has several configurations which allow visualizing and capturing all the movements of the body in real time in the computer by means of 3D avatars and graphs. It generates plain text files compatible with Excel or Matlab.

The normal patient is $174 \mathrm{~cm}$ tall and weighs $74 \mathrm{~kg}$. He was selected for his anthropometric characteristics similar to those of the amputated patient, offering a similarity in the analysis of the movement. While the amputated patient is $170 \mathrm{~cm}$ tall, and weighs $80 \mathrm{~kg}$, he uses a transtibial prosthesis (dynamic foot, suspension by pin, endoeskeletal design), he is 42 years old and have used the prosthesis for 11 years.)

The MarchaHumanaET software identifies and obtains the spatio-temporal parameters of the human gait based on the data generated by the TechNaid® equipment. This provides a flat text report of variables such as: distance, step length, step time, cadence of step, stride length, stride time and gait speed.

Fig. 1: Amputee patient, healthy patient and "MarchaHumanaET" software.
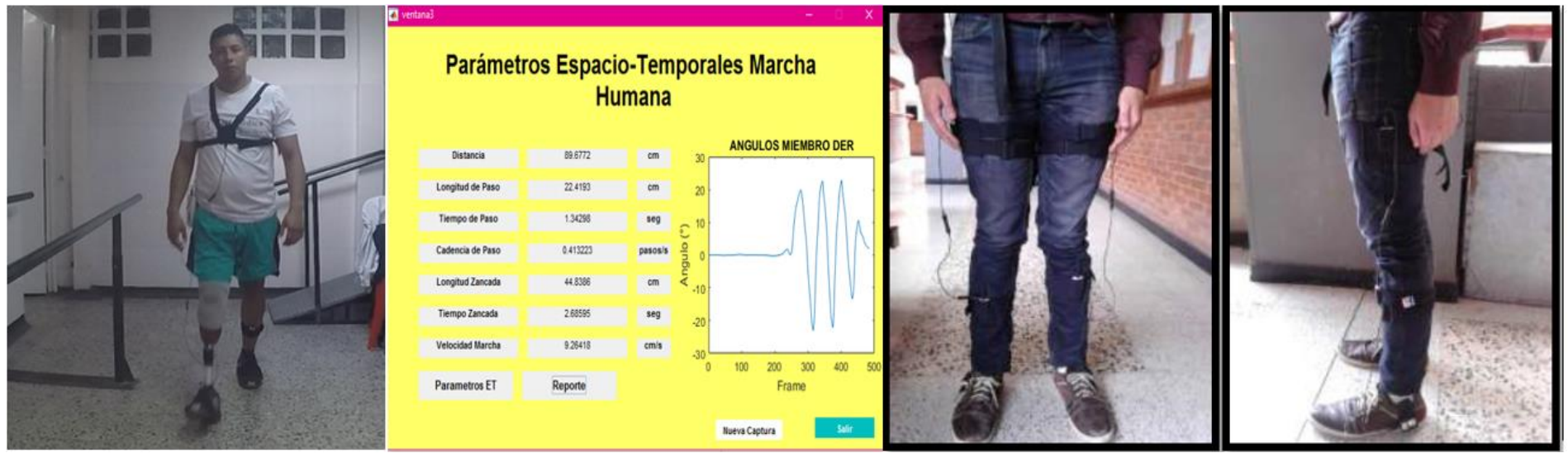

\section{Results}

The step length differs by $5 \%$, the magnitude is greater for the healthy patient data with respect to those of the amputated patient. Since this length is determined by height, the studied subjects do not vary by more than $10 \mathrm{~cm}$. [2]

The step time is not subjected to variables such as height or age of the subjects, this is determined by the speed that is present in each gait and analyzing that the gait of each patient was 50\% different is not possible to verify this parameter, but it is possible to declare that half of the healthy patient's gait speed agrees with that of the amputated patient in the same way the step time of the healthy patient was approximately double in magnitude with respect to that one of the amputated patient [3] (See Table 1).

Table 1: Spatio-temporal variables of healthy patient gait.

\begin{tabular}{|c|c|}
\hline Distance (m) & $\mathbf{5 . 9 8}$ \\
\hline Step length (m) & 0.46 \\
\hline Step time (min) & 0.03 \\
\hline Cadence of step (steps / min) & 85.5 \\
\hline Stride length (m) & 0.93 \\
\hline Stride time (s) & 3.6 \\
\hline Gait speed (m / s) & 0.57 \\
\hline
\end{tabular}

Since the cadence of step is the number of steps evident in a subject's gait, it is analyzed that the studied patients differ this data by $15 \mathrm{~cm}$ with respect to the other and also there is a difference of $6 \mathrm{~cm}$ approximately of the healthy patient compared to predetermined values by researchers [4] who present that, for the subject age, the range in which the cadence of step value should be between 91-135 (steps * min). 
Table 2: Spatio-temporal variables of the aligned socket.

\begin{tabular}{|c|c|}
\hline & Alignment \\
\hline Distance (m) & 2.123 \\
\hline Step length (m) & 0.383 \\
\hline Step time (min) & 0.020 \\
\hline Cadence of step (steps / min) & 70.89 \\
\hline Stride length (m) & 0.566 \\
\hline Stride time (s) & 2.42 \\
\hline Gait speed (m / s) & 0.248 \\
\hline
\end{tabular}

The magnitude of the parameter step length is determined by the height of the subjects and analyzing the data given by the software is slightly different from the data of the healthy patient with respect to the data of the amputated patient since their measures differ very little with each other.

\section{Discussion}

The amputated patient during the stages of the gait stands his healthy limb more on the ground with respect to the amputated limb, so that resulting force on the intact limb will be greater than that which falls on the amputated limb. This activity generates diseases in patients such as osteoporosis and osteoarthritis [5]. In the case of the amputated patient studied, these pathologies may present themselves as the spatio-temporal variables of his movement vary with respect to those of the healthy patient.

The spatio-temporal variables are important when analyzing the gait because they allow a more detailed study of the movement, presenting quantitative data can find percentage errors between values established by other studies and the analysis of the movement of the subject being studied. It is also possible to discover pathologies that may be affecting the lower limbs of an amputated patient, making comparisons between values of established spatio-temporal parameters and acquired values of movement.

The values obtained are subject to several factors such as: terrain, footwear, clothing and cargo transport, sex, age, height, physical constitution and weight of the subject. Other factors that may make the study of gait subjectively are: the personality and the emotions of the person at the time of being studied. We also found pathological factors such as: trauma, neurological pathologies, skeletal muscle, psychiatric disorders, among others.

\section{Conclusion}

It was observed that the prosthesis manages to meet the needs of the amputated joint without presenting major changes in the acquired values; the percentage of error is very low referring to the healthy patient, this indicates that the prosthesis has to be optimized in a small measure, either by the best adjustment of the socket, a better training of the patient with his prosthesis and the quality of the prosthesis (materials, measurements, stump adaptability). The prosthesis implemented in the patient is not adaptable for the different environments in the context of the patient, which causes the gait cycle is in constant change and novelties are generated by each modification of the socket constantly altering the spatio-temporal parameters according to the average.

\section{Acknowledgments}

We thank "Universidad Distrital Francisco Jose de Caldas" for its accompaniment in the studies carried out, the DIGITI research group attached to the University for the valuable monitoring of the study and to allow the use of the implemented equipment. We also thank the volunteers for allowing their body to study this research; and finally to the orthopedic and rehabilitation center for providing its spaces to acquire the measurements of each patient.

\section{References}

[1] E. Isakov, O. Keren, N. Benjuya, "Trans-tibial amputee gait: time-distance parameters and EMG activity," Prosthet Orthot Int., vol. 24, no. 3, pp. 216-20, 2000.

[2] F. Prince, D. A. Winter, G. Sjonnensen, C. Powell, R. K. Wheeldon, "Mechanical efficiency during gait of adults with transtibial amputation: a pilot study comparing the SACH, Seattle, and Golden-Ankle prosthetic feet," $J$ Rehabil Res Dev., vol. 35, no. 2, pp. 177-85, 1998.

[3] K. U. Smith, C. D. McDermid, F. E. Shideman, "Analysis of the temporal components of motion in human gait," in American Journal of Physical Medicine., no. 39, pp. 142-151, 1960. 
[4] M. P. Murray, B. Drought, R. C. Kory, "Walking Patterns of Normal Men," in The Journal of Bone And Joint Surgery., no. 46, pp. 335-360, 1964.

[5] R. L. Waters, S. Mulroy, "The energy expenditure of normal and pathological gait (Review)," in Gait and Posture., pp. 207-231, 1999. 